1 Hacettepe Journal of Mathematics and Statistics

$\bigcap$ Volume 46 (1) (2017), $91-101$

\title{
Closure operators associated with networks
}

\author{
Josef Šlapal ${ }^{* \dagger}$ and John L. Pfaltz ${ }^{\ddagger}$
}

\begin{abstract}
We study network (i.e., undirected simple graph) structures by investigating associated closure operators and the corresponding closed sets. To describe the dynamic behavior of networks, we employ continuous transformations and neighborhood homomorphisms between them. These transformations and homomorphisms are then studied. In particular, the problem of preserving generators by continuous transformations and that of preserving minimal dominating sets by neighborhood homomorphisms are dealt with.
\end{abstract}

Keywords: Network, Closure operator, Transformation.

2000 AMS Classification: 05C82, 54A05, 54C10, 90B18.

\section{Introduction}

Networks are ubiquitous in science and engineering, cf. [12] for a bibliography of more than 400 network applications. Network structures are invariably described in combinatorial terms, that is, numerically. Scientists typically count and measure. This paper, instead, uses the closed sets of an associated closure operator to define the structure of a network. Like open sets in continuous manifolds, closed sets can be a powerful tool for analyzing the structure of discrete systems.

In economics, closure is associated with rational choice operators $[8,11,10]$. The Galois closure can be used to extract rules from data sets for subsequent usage in artificial intelligence reasoning systems $[16,17]$. If a system can be partially (or totally) ordered, then the closed sets are usually intervals, ideals or filters $[7,9]$. In this paper, we apply closed sets to the structure of undirected graphs representing networks.

*IT4Innovations Centre of Excellence, Brno University of Technology,

Email: slapal@fme.vutbr.cz

$\dagger^{\dagger}$ Corresponding Author.

${ }^{\ddagger}$ Dept. of Computer Science, University of Virginia,

Email: jlp@virginia.edu

Doi : 10.15672/HJMS.2016.397 
Since networks change over time [2, 18], this paper also seeks a mechanism for describing such changes. We examine the properties of smooth, or continuous, transformations of networks [14]. The question is, what changes to the structure of the network, as revealed by its closure properties, must ensue. We show, among others, that continuous transformations preserve generating sets. Another kind of network transformations discussed are neighborhood homomorphisms, which are shown to preserve dominating sets. Their special cases, the strong homomorphisms, even preserve the minimal dominating sets under certain conditions.

Throughout the paper, we will use the suffix denotation for mappings to avoid multiple parentheses and emphasize the importance of the domain elements. Thus, given a mapping $f: X \rightarrow Y(X, Y$ sets $)$ and a point $x \in X$, we write $x . f$ to denote the $f$-image of $x$. Similarly, if $g: Y \rightarrow Z$ is another mapping, we write $f . g$ to denote the composition of $f$ and $g$. Hence, $x . f . g$ denotes the $f . g$-image of $x$. We will also often simplify the denotation of sets given by enumerating their elements - we will omit the commas between the elements and, in many cases, also the curly brackets. Thus, for example, $\{a b c d\}$ or $a b c d$ will be used for short rather than $\{a, b, c, d\}$ (we will delimit a set with the curly brackets only if we want to emphasize its set nature). In particular, we will usually not distinguish notationally between elements and singleton subsets of a set.

\section{Networks and neighborhood closure operators}

Let $\mathcal{N}=(S, \rho)$ be a set $S$ of points, elements or nodes together with an $a d$ jacency relation, i.e., a reflexive and symmetric binary relation $\rho$ on $S$. (Note that, although in the literature, adjacency relations are usually defined to be irreflexive and symmetric, our definition of adjacency relations causes no confusion because there is a bijection between the reflexive and irreflexive binary relations on a set.) We then call $\mathcal{N}$ a network. For any subset $Y \subseteq S$, we put $Y . \rho=\{x \mid \exists y \in Y:(x, y) \in \rho\}$. The set $Y . \rho$ is called the neighborhood of $Y$. Clearly, $\emptyset . \rho=\emptyset$ and $x \in y . \rho \Leftrightarrow y \in x . \rho$ whenever $x, y \in S$. Next, we have $X \subseteq X . \rho=\bigcup_{x \in X} x . \rho$ for every $X \subseteq S$. It follows that $X \subseteq Y \Rightarrow X . \rho \subseteq Y . \rho$ and $(X \cup Y) . \rho=X . \rho \cup Y$. $\rho$ for all $X, Y \subseteq S$. Clearly, we also have $S . \rho=S$.

Given a network $\mathcal{N}=(S, \rho)$ and subsets $X, Y \subseteq S, X$ is said to dominate $Y$ in $\mathcal{N}$ if $Y \subseteq X . \rho$.

We can represent a network $(S, \rho)$ as an undirected simple graph with the vertex set $S$ and each edge being a two-element subsets $\{x, y\} \subseteq S$ with $(x, y) \in \rho$ (or, equivalently, $(y, x) \in \rho$ ). The neighborhood of any point is then the set of those vertices that are adjacent to the point in the graph. For example, in the network $(S, \rho)$ with $S=\{a b c d e f g h\}$ representd by the undirected graph in Figure 1, we have $\{a\} . \rho=\{a, b, c\}$ or, more simply, a. $\rho=a b c$. Clearly, $\{a\}$ dominates $\{a b c\}$ and $\{c h\}$ dominates $S$. There is a large literature on dominating sets in undirected networks, c.f. $[5,6]$.

When studying the structure of a network $(S, \rho)$, we found it to be advantageous to consider a convenient associated operator on $S$. In this note, to be able to apply topological methods in the study of network structure, we employ a closure operator. Let us recall first the definition of a closure operator. 


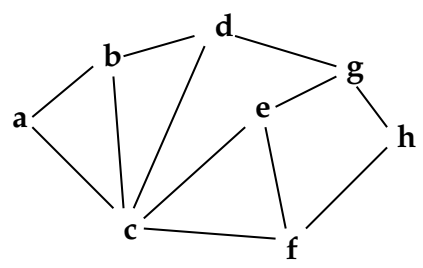

Figure 1. An undirected graph.

Let $S$ be a set. A mapping $u: 2^{S} \rightarrow 2^{S}$ (where $2^{S}$ denotes the power set of $S$ ) is said to be a closure operator on $S$ (cf. [15]) if, for all $Y, Z \subseteq S$,

(C1) $Y \subseteq Y . u$ (extensivity),

(C2) $Y \subseteq Z \Rightarrow Y . u \subseteq Z . u$ (monotonicity), and,

(C3) Y.u.u $=Y . u$ (idempotency).

A subset $Y \subseteq S$ is closed if $Y=Y$.u. A subset $X$ of a closed set $Y \subseteq S$ is said to generate $Y$ if $X . u=Y$.

It is well known that the intersection of closed sets must be closed so that a closure operator $u$ on $S$ is uniquely determined by the system of closed sets. Indeed, for every subset $Y \subseteq S$, we have $Y$.u $=\bigcap\{Z \mid Z$ closed and $Y \subseteq Z\}$.

By (C1), the set $S$ must be closed. The empty set may or may not be closed. If it is, then $u$ is said to be grounded.

By a closure system we mean a pair $(S, u)$ with $S$ a set (of points or elements) and $u$ a closure operator on $S$.

Let $\mathcal{N}=(S, \rho)$ be a network and $Y \subseteq S$ a subset. Then, we define the neighborhood closure of $Y$ to be the subset $Y . u \rho \subseteq S$ given as follows:

$$
Y . u \rho=\{x \in S \mid x . \rho \subseteq Y . \rho\} .
$$

Clearly, for all $Y \subseteq S, Y . u_{\rho} \subseteq Y . \rho$ and $Y . u_{\rho} . \rho=Y . \rho$.

Assigning to every subset of $S$ its neighborhood closure, we get a mapping $u_{\rho}: 2^{S} \rightarrow 2^{S}$.

2.1. Proposition. $u_{\rho}$ is a grounded closure operator on $S$.

Proof. Clearly, $u_{\rho}$ is grounded and extensive by definition.

Let $X, Y \subseteq S$ be subsets, $X \subseteq Y$, and let $z \in X . u \rho$. Then, $z . \rho \subseteq X . \rho \subseteq Y . \rho$, hence, $z \in Y$.u. Therefore, $u_{\rho}$ is monotone.

Let $X \subseteq S$ be a subset and let $z \in Y . u_{\rho} . u_{\rho}$. Then, $z . \rho \subseteq Y . u_{\rho} . \rho=\bigcup_{x \in Y . u} x \cdot \rho \subseteq$ $\bigcup_{x \in Y} x . \rho=Y . \rho$, hence, $z \in Y . u \rho$. Thus, $u_{\rho}$ is idempotent and the proof is complete.

2.2. Definition. The closure operator $u_{\rho}$ and the closure system $\left(S, u_{\rho}\right)$ are said to be the neighborhood closure operator and the neighborhood closure system, respectively, associated with $(S, \rho)$.

2.3. Remark. If, in the definition of a grounded closure operator $v$ on a set $S$, we replace the axiom (C3) of idempotency by the axiom of additivity, i.e., $v(X \cup Y)=v X \cup v Y$ whenever $X, Y \subseteq S$, we get a so-called pretopology $v$ on $S$ (and a pretopological space $(S, v)$ ) - cf. [20]. A neighborhood of a subset $X \subseteq S$ in 
a pretopological space $(S, v)$ is any subset $Y \subseteq S$ such that $X \cap(S-Y) \cdot v=\emptyset$. There is a natural way of associating a pretopology $v_{\rho}$ on a set $S$ with any reflexive binary relation $\rho$ on $S$ : we put $X . v_{\rho}=\{x \in S \mid \exists y \in X:(x, y) \in \rho\}$ for every $X \subseteq S$ - see [21]. Let $X \subseteq S$. If $\rho$ is a reflexive and symmetric binary relation on $S$, then $X . v_{\rho}=X . \rho$. But then, $X . \rho$ is also the smallest neighborhood (with respect to set inclusion) of $X$ in the closure space $\left(S, v_{\rho}\right)$. This fact justifies our calling $X . \rho$ the neighborhood of $X$.

2.4. Proposition. For every pair of sets $X, Y \subseteq S, X . u_{\rho} \subseteq Y . u_{\rho}$ if and only if $X . \rho \subseteq Y . \rho$.

Proof. Let $X, Y \subseteq S$ be subsets with $X . u_{\rho} \subseteq Y . u_{\rho}$. Let $x \in X . u_{\rho}$ implying $x \in y . u_{\rho}$ for some $y \in X \subseteq X . u_{\rho} \subseteq Y . u_{\rho}$. Then, $y . \rho \subseteq Y$. $\rho$ so that $x \in Y . \rho$. Consequently, $X . \rho \subseteq Y . \rho$.

To prove the converse inclusion, suppose that $X . \rho \subseteq Y . \rho$. Let $z \in X . u_{\rho}$, implying $z . \rho \subseteq X . \rho \subseteq Y . \rho$. Then, $z \in Y . u_{\rho}$ and, therefore, $X . u_{\rho} \subseteq Y . u_{\rho}$.

As an immediate consequence of Proposition 2.4 we get

2.5. Corollary. For every pair of sets $X, Y \subseteq S, X . u_{\rho}=Y . u_{\rho}$ if and only if $X . \rho=Y . \rho$.

One might expect that every point in a discrete space must be closed with respect to the neighborhood closure. But this need not be true, as shown in Figure 1, where c. $\rho=a b c d e f, a . \rho=a b c \subseteq c . \rho$ and $b . \rho=a b c d \subseteq c . \rho$, while $d . \rho=b c d g \nsubseteq c . \rho, e . \rho=c e f g \nsubseteq c . \rho$, and $f . \rho=c e f h \nsubseteq c . \rho$, so that $\{c\} . u_{\rho}=\{a b c\}$.

2.6. Proposition. Let $X, Y \subseteq S$ be subsets, $X \subseteq Y$ and $Y$ closed in $\left(S, u_{\rho}\right)$. Then, $X$ generates $Y$ in $\left(S, u_{\rho}\right)$ if and only if $X . \rho=Y . \rho$.

Proof. Let $X$ generate $Y$, i.e., let $X . u_{\rho}=Y$. Since $X . u_{\rho} . \rho=X . \rho$, we have $Y . \rho=X . \rho$.

Conversely, let $X . \rho=Y . \rho$ and let $y \in Y$. Then, $y . \rho \subseteq Y . \rho=X . \rho$, so that $y \in X . u_{\rho}$. Therefore, we have $Y \subseteq X . u_{\rho}$. As the converse inclusion is obvious, the proof is complete.

Let $(S, u)$ be a closure system. Then, it is useful to deal with the structure of closed sets because closed sets uniquely determine the closure operator $u$. In the case of a neighborhood closure operator associated with a network, the structure of closed sets may be regarded as the structure of the network. The closed sets may be partially ordered by inclusion so that they create a complete lattice, $\mathcal{L}_{u}$, in which infima coincide with intersections - see [15] for more details.

The neighborhood closure lattice $\mathcal{L}_{u}$ corresponding to the neighborhood closure operator associated with the network of Figure 1 is shown in Figure 2. This lattice has none of the regular structures one usually sees in textbook examples. Nevertheless, it carries considerable information.

\section{Network transformations}

Let $S$ and $S^{\prime}$ be sets. By a transformation $S \stackrel{f}{\longrightarrow} S^{\prime}$ (between $S$ and $S^{\prime}$ ) we mean a mapping $f: 2^{S} \rightarrow 2^{S^{\prime}}$. Given a mapping $f: S \rightarrow S^{\prime}$, its extension is the 


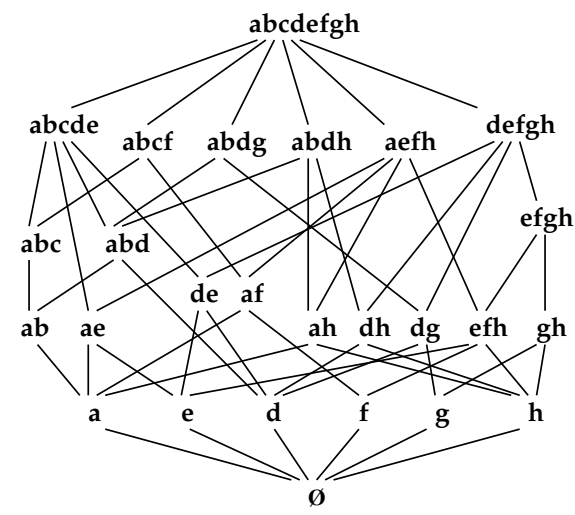

Figure 2. Neighborhood closure lattice of Figure 1.

transformation $S \stackrel{f^{+}}{\longrightarrow} S^{\prime}$ defined by $Y . f^{+}=\{f(y) \mid y \in Y\}$ for every $Y \subseteq S$ (in the literature, $f^{+}$is often said to be lifted from $f$ ). As usual, we will write $f$ instead of $f^{+}$so that a mapping and its extension will be denoted by the same symbol it will always be clear from the context whether $f$ means a mapping $f: S \rightarrow S^{\prime}$ or its extension $S \stackrel{f}{\longrightarrow} S^{\prime}$. Of course, there can be many transformations $S \stackrel{f}{\longrightarrow} S^{\prime}$ other than those extended from mappings $f: S \rightarrow S^{\prime}$.

A transformation $S \stackrel{f}{\longrightarrow} S^{\prime}$ is said to be monotone if, whenever $X, Y \subseteq S$, $X \subseteq Y$ implies $X . f \subseteq Y$.f. Note that a transformation that has been extended from a mapping must be monotone.

Let $S \stackrel{f}{\longrightarrow} S^{\prime}$ be a transformation and $Z \subseteq S^{\prime}$ a subset. If the set of all subsets $Y \subseteq S$ with $Y . f=Z$ is nonempty and has a greatest element (with respect to set inclusion), then the greatest element is said to be the inverse image of $Z$ under $f$. Thus, if every subset of $S^{\prime}$ has an inverse image, we get a transformation between $S^{\prime}$ and $S$ assigning to every subset of $S^{\prime}$ its inverse image under $f$. This transformation will be denoted by $S^{\prime} \stackrel{f^{-1}}{\longrightarrow} S$. Clearly, if $S \stackrel{f}{\longrightarrow} S^{\prime}$ is an extension of a mapping, then every subset of $S^{\prime}$ has an inversion.

Let $(S, \rho)$ and $\left(S^{\prime}, \rho^{\prime}\right)\left((S, u)\right.$ and $\left.\left(S^{\prime}, u^{\prime}\right)\right)$ be networks (closure systems) and let $S \stackrel{f}{\longrightarrow} S^{\prime}$ be a transformation. We then write $(S, \rho) \stackrel{f}{\longrightarrow}\left(S^{\prime}, \rho^{\prime}\right)\left((S, u) \stackrel{f}{\longrightarrow}\left(S^{\prime}, u^{\prime}\right)\right)$ and say that $f$ is a transformation between $(S, \rho)$ and $\left(S^{\prime}, \rho^{\prime}\right)\left((S, u)\right.$ and $\left.\left(S^{\prime}, u^{\prime}\right)\right)$.

3.1. Definition. A transformation $(S, \rho) \stackrel{f}{\longrightarrow}\left(S^{\prime}, \rho^{\prime}\right)$ between networks is said to be neighborhood monotone if, whenever $X, Y \subseteq S$,

$$
X . \rho \subseteq Y . \rho \Rightarrow X . f . \rho^{\prime} \subseteq Y . f . \rho^{\prime} .
$$

Note that a transformation that is monotone need not be neighborhood monotone, and vice versa.

3.2. Definition. ([13, 14, 21]) A transformation $\left.(S, u) \stackrel{f}{\longrightarrow}\left(S^{\prime}, u^{\prime}\right)\right)$ between closure systems is said to be continuous if, whenever $Y \subseteq S$,

$$
\text { Y.u.f } \subseteq \text { Y.f.u'. }
$$


A transformation $(S, \rho) \stackrel{f}{\longrightarrow}\left(S^{\prime}, \rho^{\prime}\right)$ between networks is called continuous if the transformation $\left(S, u_{\rho}\right) \stackrel{f}{\longrightarrow}\left(S^{\prime}, u_{\rho^{\prime}}\right)$ between neighborhood closure systems is continuous.

3.3. Proposition. Let $(S, u) \stackrel{f}{\longrightarrow}\left(S^{\prime}, u^{\prime}\right)$ be a monotone transformation between closure systems. If $f$ is continuous, then $Y$.u. $f=Y$.f for every subset $Y \subseteq S$ with $Y$.f closed in $\left(S^{\prime}, u^{\prime}\right)$. The converse is true provided that every subset of $S^{\prime}$ has an inverse image under $f$ and the transformation $S^{\prime} \stackrel{f^{-1}}{\longrightarrow} S$ is monotone.

Proof. Let $f$ be continuous and let $Y \subseteq S$ be a subset with $Y$.f closed in $\left(S^{\prime}, u^{\prime}\right)$. Then, Y.u.f $\subseteq Y . f . u^{\prime}=Y . f$. The converse inclusion follows from the extensiveness of $u$ and monotonicity of $f$.

Conversely, let Y.u.f $=Y . f$ for every subset $Y \subseteq S$ with $Y . f$ closed in $\left(S^{\prime}, u^{\prime}\right)$ and let every subset of $S^{\prime}$ have an inverse image under $f$. Let $X \subseteq S$ be an arbitrary subset. Then, X.f. $u^{\prime}$ is closed in $\left(S^{\prime}, u^{\prime}\right)$ and $X . f . u^{\prime}=X . f . u^{\prime} . f^{-1} . f$. Therefore, $X . f . u^{\prime} . f^{-1} . u . f=X . f . u^{\prime}$. Next, since $X . f \subseteq X . f . u^{\prime}$, we have $X \subseteq X . f . f^{-1} \subseteq$ $X . f . u^{\prime} . f^{-1}$. Hence, $X . u \subseteq X . f . u^{\prime} . f^{-1} . u$, which yields $X . u . f \subseteq X . f . u^{\prime} . f^{-1} . u . f$. Consequently, X.u.f $\subseteq X . f . u^{\prime}$ and the continuity of $f$ is proved.

3.4. Corollary. Let $(S, u) \stackrel{f}{\longrightarrow}\left(S^{\prime}, u^{\prime}\right)$ be a monotone transformation between closure systems. If $f$ is continuous, then, for every closed subset $Z$ of $\left(S^{\prime}, u^{\prime}\right)$, the inverse image of $Z$ under $f$ (if it exists) is closed in $(S, u)$. The converse is true provided that every subset of $S^{\prime}$ has an inverse image under $f$ and the transformation $S^{\prime} \stackrel{f^{-1}}{\longrightarrow} S$ is monotone.

Proof. Let $f$ be continuous and let $Z$ be a closed subset of $\left(S^{\prime}, u^{\prime}\right)$ having an inverse $Y$ image under $f$. Since $Y . u . f=Y$.f by Proposition 3.3, we have $Y . u \subseteq Y$. Therefore, $Y$ is closed in $(S, u)$.

Conversely, suppose that every subset of $S^{\prime}$ has an inverse image under $f$ and the transformation $S^{\prime} \stackrel{f^{-1}}{\longrightarrow} S$ is monotone. Let, for every closed subset $Z$ of $\left(S^{\prime}, u^{\prime}\right)$, the inverse image of $Z$ under $f$ (if it exists) is closed in $(S, u)$ and let $Y \subseteq S$ be an arbitrary subset with $Y . f$ closed in $\left(S^{\prime}, u^{\prime}\right)$. Then, $Y . f . f^{-1} . u=Y . f . f^{-1}$ because $Y . f . f^{-1}$ is closed in $(S, u)$. Since $Y \subseteq Y . f . f^{-1}$, we have Y.u.f $\subseteq Y . f . f^{-1} . u . f=$ $Y . f . f^{-1} . f=Y . f$. As the converse inclusion is evident (it follows from the extensiveness of $u$ and monotonicity of $f$ ), we have Y.u.f $=Y . f$. Therefore, $f$ is continuous by Proposition 3.3.

It is well known that, for the transformations between closure systems that are extensions of mappings, also the converse of Corollary 3.4 is true.

3.5. Proposition. If $(S, \rho) \stackrel{f}{\longrightarrow}\left(S^{\prime}, \rho^{\prime}\right)$ is a monotone and continuous transformation between networks, then Y.u.f.u' $=Y . f . u^{\prime}$.

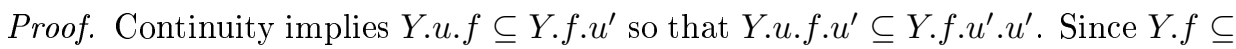
$Y . u$.f by monotonicity of $u$ and $f$, we get $Y . f . u^{\prime} \subseteq Y . u . f . u^{\prime}$.

3.6. Proposition. Let $(S, u) \stackrel{f}{\longrightarrow}\left(S^{\prime}, u^{\prime}\right),\left(S^{\prime}, u^{\prime}\right) \stackrel{g}{\longrightarrow}\left(S^{\prime \prime}, u^{\prime \prime}\right)$ be transformations and let $g$ be monotone. If both $f$ and $g$ are continuous, then so is $S \stackrel{f \cdot g}{\longrightarrow} S^{\prime \prime}$. 
Proof. We have X.u.f $\subseteq X . f . u^{\prime}$ for any $X \subseteq S$ and $Y . u^{\prime} . g \subseteq Y . g . u^{\prime \prime}$ for any $Y \subseteq S^{\prime}$. Consequently, as $g$ is monotone, X.u.f.g $\subseteq X . f . u^{\prime} . g \subseteq X . f . g . u^{\prime \prime}$. This means that $f . g$ is continuous.

In his seminal work [13], Ore considered only extended, continuous transformations.

3.7. Proposition. Let $(S, \rho) \stackrel{f}{\longrightarrow}\left(S^{\prime}, \rho^{\prime}\right)$ be a monotone transformation between networks. Then $f$ is continuous if and only if $f$ is neighborhood monotone.

Proof. Let $f$ be continuous and let $X, Y \subseteq S$ be subsets with $X . \rho \subseteq Y$. $\rho$. By Proposition 2.4, $X \subseteq X . u_{\rho} \subseteq Y . u_{\rho}$. Thus, $X . f \subseteq Y . u_{\rho} . f \subseteq Y . f . u_{\rho^{\prime}}$ by continuity.

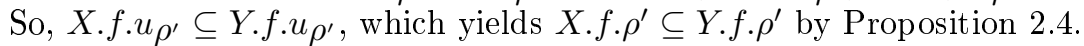

Conversely, let $f$ be neighborhood monotone and let $Y \subseteq S$ be a subset. Since $Y . u \rho . \rho=Y . \rho$, we have Y.u $\rho . f . \rho^{\prime} \subseteq Y . f . \rho^{\prime}$ by neighborhood monotinicity. Thus, by Proposition 2.4, Y.u $\rho . f . u_{\rho^{\prime}} \subseteq Y$.f.u $\rho_{\rho^{\prime}}$. Now, by the extensivity of $u^{\prime}, Y . u . f \subseteq$ Y.u.f.u $\subseteq$ Y.f.u'.

3.8. Proposition. Let $(S, \rho) \stackrel{f}{\longrightarrow}\left(S^{\prime}, \rho^{\prime}\right)$ be a monotone and continuous transformation between networks. If $X$ generates $Z$ in $\left(S, u_{\rho}\right)$ and $Z . f$ is closed in $\left(S^{\prime}, u \rho^{\prime}\right)$, then $X$.f generates $Z$.f in $\left(S^{\prime}, u \rho^{\prime}\right)$.

Proof. Let $X$ generate $Z$ in $(S, u \rho)$. Then, $X . \rho=Z$. $\rho$ by Proposition 2.6. Since $f$ is continuous, $f$ is neighborhood monotone by Proposition 3.7 and we have $X . f . \rho=Z . f . \rho$. Therefore, X.f generates $Z . f$ in $\left(S^{\prime}, u_{\rho^{\prime}}\right)$ by Proposition 2.6.

3.9. Definition. A transformation $(S, u) \stackrel{f}{\longrightarrow}\left(S^{\prime}, u^{\prime}\right)$ between closure systems is said to be closed if $Y$. $f$ is closed in $\left(S^{\prime}, u^{\prime}\right)$ whenever $Y$ is closed in $(S, u)$.

3.10. Proposition. Let $(S, u) \stackrel{f}{\longrightarrow}\left(S^{\prime}, u^{\prime}\right)$ be a transformation between closure systems. If Y.f. $u^{\prime} \subseteq Y$.u.f for all $Y \subseteq S$, then $f$ is closed. The converse is true provided that $f$ is monotone.

Proof. Let $Y . f . u^{\prime} \subseteq Y$.u.f for every subset $Y \subseteq S$. If $Y$ is closed, then $Y$.f. $u^{\prime} \subseteq Y$.f, so that $Y$. $f$ is closed. Hence, $f$ is closed.

Conversely, let $f$ be monotone and closed and let $Y \subseteq S$ be a subset. Then, Y.f. $u^{\prime} \subseteq$ Y.u.f. $u^{\prime}=$ Y.u.f because Y.u.f is closed in $\left(S^{\prime}, u^{\prime}\right)$ (as Y.u is closed in $(S, u))$.

3.11. Definition. A transformation $(S, \rho) \stackrel{f}{\longrightarrow}\left(S^{\prime}, \rho^{\prime}\right)$ between networks is said to be a homomorphism if, for every $Y \subseteq S$,

$$
Y . \rho . f \subseteq Y . f . \rho^{\prime} .
$$

Homomorphisms are common in graph theory (and, more generally, theory of binary relations - cf. [19]). If $G=(S, \mathcal{E})$ and $G^{\prime}=\left(S^{\prime}, \mathcal{E}^{\prime}\right)$ are undirected graphs with edge sets $\mathcal{E}$ and $\mathcal{E}^{\prime}$, then a mapping $f: S \rightarrow S^{\prime}$ is said to be a graph homomorphism if $\{x, y\} \in \mathcal{E}$ implies $\{f(x), f(y)\} \in \mathcal{E}^{\prime}[1,3,4]$. The above definition of homomorphisms is obtained by naturally extending the definition of graph homomorphisms. 
Homomorphisms need not be continuous even if they are monotone. Consider the monotone homomorphism $(S, \rho) \stackrel{f}{\longrightarrow}\left(S^{\prime}, \rho^{\prime}\right)$ displayed in Figure 3, which is an extension of the mapping $f:\left\{x, y_{1}, y_{2}, z\right\} \rightarrow\left\{x^{\prime}, y^{\prime}, z^{\prime}\right\}$ given by $x . f=x^{\prime}$, $y_{1} . f=y_{2} . f=y^{\prime}$, and $z \cdot f=z^{\prime}$. So, $x \cdot u_{\rho}=x y_{1}$ and $z \cdot u_{\rho}=y_{2} z$ while $x^{\prime} \cdot u_{\rho^{\prime}}=x^{\prime}$ and $z^{\prime} \cdot u_{\rho^{\prime}}=z^{\prime}$. Thus, $\{x\} \cdot u_{\rho} \cdot f=\left\{x^{\prime} y^{\prime}\right\} \nsubseteq\left\{x^{\prime}\right\}=\{x\} . f \cdot u_{\rho^{\prime}}$.

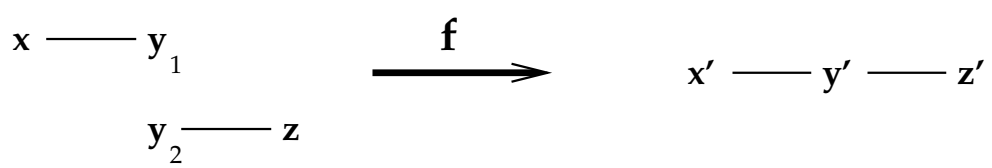

Figure 3. A homomorphism that is not continuous.

Monotone and continuous transformations, too, need not be homomorphisms. Consider the monotone, continuous transformation $(S, \rho) \stackrel{f}{\longrightarrow}\left(S^{\prime}, \rho^{\prime}\right)$ displayed in Figure 4, which is an extension of the bijection $f:\{w, x, y, z\} \rightarrow\left\{w^{\prime}, x^{\prime}, y^{\prime}, z^{\prime}\right\}$ given by $t . f=t^{\prime}$ for every $t \in\{w, x, y, z\}$.

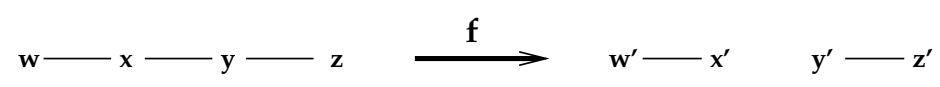

Figure 4. A monotone, continuous map that is not a homomorphism.

Clearly, $\{w x y\}=\{w x y\} . f=\{x\} . \rho . f \nsubseteq\{x\} . f . \rho^{\prime}=\left\{w^{\prime} x^{\prime}\right\}$, but it is easy to verify that $f$ is continuous because the closed sets in $(S, \rho)$ and $\left(S^{\prime}, \rho^{\prime}\right)$ are $\{\emptyset, w x y z, w x, y z, w, z\}$ and $\left\{\emptyset, w^{\prime} x^{\prime} y^{\prime} z^{\prime}, w^{\prime} x^{\prime}, y^{\prime} z^{\prime}\right\}$, respectively

3.12. Proposition. Let $(S, \rho) \stackrel{f}{\longrightarrow}\left(S^{\prime}, \rho^{\prime}\right)$ be a monotone homomorphism between networks. If $X$ dominates $Y$ in $S$, then $X$.f dominates $Y$.f in $S^{\prime}$.

Proof. If $Y \subseteq X$. $\rho$, then $Y . f \subseteq X . \rho . f \subseteq X . f . \rho^{\prime}$

3.13. Definition. A transformation $(S, \rho) \stackrel{f}{\longrightarrow}\left(S^{\prime}, \rho^{\prime}\right)$ between networks is called a strong homomorphism if, for every $Y \subseteq S$,

$$
Y . \rho . f=Y . f \cdot \rho^{\prime} .
$$

Effectively, if $\left(x^{\prime}, y^{\prime}\right) \in \rho^{\prime}$ and $x \in S$ is a point with $x . f=x^{\prime}$, then there exists $y \in S$ such that $y . f=x^{\prime}$ and $\{x, y\} \in \rho$ is an edge in $S$. The transformation in Figure 5 represents the typical configuration of a strong homomorphism. The transformation is an extension of the mapping $f:\left\{x_{1}, x_{2}, y_{1}, y_{2}, z_{1}, z_{2}\right\} \rightarrow\left\{x^{\prime}, y^{\prime}, z^{\prime}\right\}$ given by $x_{1} . f=x_{2} . f=x^{\prime}, y_{1} \cdot f=y_{2} . f=y^{\prime}$, and $z_{1} \cdot f=z_{2} . f=z^{\prime}$.

3.14. Proposition. Let $(S, \rho) \stackrel{f}{\longrightarrow}\left(S^{\prime}, \rho^{\prime}\right)$ be a strong homomorphism between networks that is an extension of a mapping. Then,

(a) $f$ is continuous,

(b) $f$ is closed provided that, for every $X \subseteq S, x \in X$. $\rho$ and $x . f \subseteq X$.f imply 


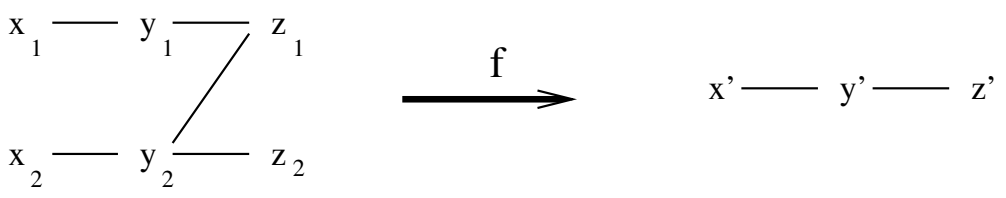

Figure 5. Typical configuration for a neighborhood homomorphism.

$x \in X$, and

(c) $Y^{\prime} \cdot f^{-1} \cdot \rho \subseteq Y^{\prime} \cdot \rho^{\prime} \cdot f^{-1}$.

Proof. (a) Let $Y \subseteq S$ be a subset and $z^{\prime} \in Y$.up. $f$ a point. Then, there exists $z \in$ $Y . u \rho$ with $z . f=z^{\prime}$. Thus, $z . \rho \subseteq Y . \rho$ and $z^{\prime} . \rho^{\prime}=z . f . \rho^{\prime}=z . \rho . f \subseteq Y . \rho . f=Y . f . \rho^{\prime}$.

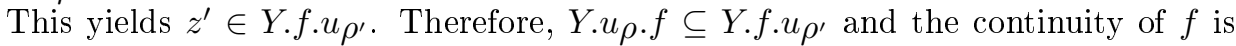
proved.

(b) Let $Y \subseteq S$ be a subset and $z^{\prime} \in Y$.f.u $\rho_{\rho^{\prime}}$ a point. Then, $z^{\prime} . \rho \subseteq Y . f . \rho$. Since $z^{\prime} \in Y . f . u_{\rho^{\prime}} \subseteq Y . f . \rho=Y . \rho . f$, there exists $z \in Y . \rho$ such that $z^{\prime}=z . f$. Let $x \in z . \rho \subseteq Y . \rho . \rho$. Then, $x . f \subseteq z . \rho . f=z . f . \rho^{\prime}=z^{\prime} . \rho^{\prime} \subseteq Y . f . \rho^{\prime}=Y . \rho . f$. Because of the extra provision, $x \in Y$. $\rho$. Thus, $z . \rho \subseteq Y . \rho$, i.e., $z \in Y . u_{\rho}$. Hence, $z^{\prime} \subseteq Y . u \rho . f$. Therefore, Y.f. $u_{\rho^{\prime}} \subseteq Y . u \rho . f$ and the closedness of $f$ is proved.

(c) Let $Y \subseteq S^{\prime}$ be a subset. Then, $Y . f^{-1} . \rho \subseteq Y . f^{-1} \cdot \rho . f . f^{-1}=Y . f^{-1} \cdot f \cdot \rho^{\prime} . f^{-1}=$ $Y . \rho^{\prime} . f^{-1}$. Conversely, let $z \in Y . \rho^{\prime} . f^{-1}$ be a point and put $z^{\prime}=z . f=\in Y^{\prime} . \rho^{\prime}$. Then, there exists $y \in Y$ with $z^{\prime} \in y$. $\rho^{\prime}$, i.e., with $y \in z^{\prime} . \rho^{\prime}$. Now, since $f$ is a

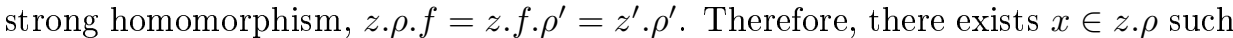
that $x . f=y \in Y$. Thus, we have $z \in x . \rho$ and $x \in y . f^{-1} \subseteq Y . f^{-1}$. Therefore, $z \in Y . f^{-1} . \rho$ and the inclusion $Y^{\prime} . \rho^{\prime} . f^{-1} \subseteq Y^{\prime} . f^{-1} . \rho$ is proved.

In contrast to monotone homomorphisms, which may not be continuous (see Figure 3), monotone strong homomorphisms always are.

We have seen that the domination is preserved by monotone homomorphisms (Proposition 3.12). It would be convenient if minimal dominating sets were preserved by (monotone) strong homomorphisms. Unfortunately, this need not be true as illustrated by the strong homomorphism displayed in Figure 6, which is an extension of the mapping $f:\{a, b, c, d, e, f, g, h\} \rightarrow\left\{a^{\prime}, b^{\prime}, e^{\prime}, g^{\prime}\right\}$ given by $a . f=a^{\prime}$, $b . f=c . f=d . f=b^{\prime}$, e.f $=f . f=e^{\prime}$, and $g . f=h . f=g^{\prime}$. The minimal dominating sets of $S$ and $S^{\prime}$ are enumerated. Here, aeh is a minimal dominating set of $S$, but $a^{\prime} e^{\prime} g^{\prime}$, although dominating in $S^{\prime}$, is not minimal. Only the minimal dominating sets aef, agh, bef, and $c g$ are mapped onto the minimal dominating sets $a^{\prime} e^{\prime}, a^{\prime} g^{\prime}$, $b^{\prime} e^{\prime}$, and $b^{\prime} g^{\prime}$, respectively.

3.15. Proposition. Let $(S, \rho) \stackrel{f}{\longrightarrow}\left(S^{\prime}, \rho^{\prime}\right)$ be a strong homomorphism that is an extension of a mapping. Let $X$ be a minimal dominating set of $Y$ in $(S, \rho)$ such that, for every element $x \in Y . \rho, x^{\prime} \in X . f$ and $x^{\prime}=x . f \in X . f$ imply $x \in X$. Then, $X . f$ is a minimal dominating set of $Y$.f in $\left(S^{\prime}, \rho^{\prime}\right)$.

Proof. Put $X^{\prime}=X . f$ and $Y^{\prime}=Y$.f. By Proposition 3.12, $X^{\prime}$ dominates $Y^{\prime}$, i.e., $Y^{\prime} \subseteq X^{\prime}$. $\rho^{\prime}$. If $Y^{\prime}=\emptyset$, then $Y=\emptyset$, so that $x=\emptyset$. Hence, $X^{\prime}=\emptyset$ and, consequently, $X^{\prime}$ is a minimal dominating set of $Y^{\prime}$ in $\left(S^{\prime}, \rho^{\prime}\right)$. Let $Y^{\prime} \neq \emptyset$ and 


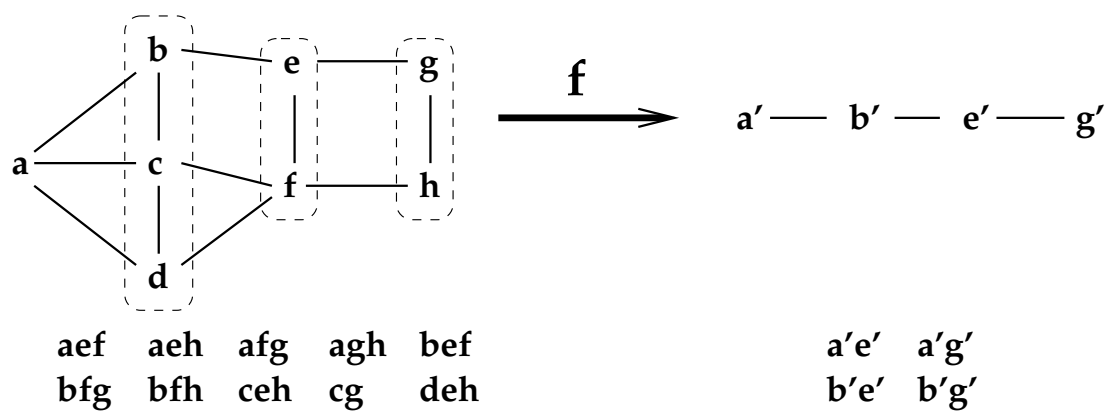

Figure 6. Neighborhood homomorphisms may not preserve minimal dominating sets.

suppose that $X^{\prime}$ is not a minimal dominating set of $Y^{\prime}$. Then, there exists a proper non-empty subset $X_{1}^{\prime} \subset X^{\prime}$ dominating $Y^{\prime}$, i.e., fulfilling $Y^{\prime} \subseteq X_{1}^{\prime} . \rho^{\prime}$. Let $x_{0}^{\prime} \in$ $X^{\prime}-X_{1}^{\prime}$ be an arbitrary element. Since $X_{1}^{\prime} \subseteq X^{\prime}-x_{0}^{\prime}$, we have $Y^{\prime} \subseteq\left(X^{\prime}-x_{0}^{\prime}\right) . \rho^{\prime}$. Let $x_{0} \in X$ be a point with $x_{0}^{\prime}=x_{0} . f$ and put $Y_{0}^{\prime}=Y^{\prime} \cap x_{0}^{\prime} . \rho^{\prime}$. Let $y \in Y$ be an arbitrary element and put $y^{\prime}=y$. $f$. Then, $y^{\prime} \in Y^{\prime}$.

If $y^{\prime} \notin Y_{0}^{\prime}$, then $y$. $f \notin x_{0}^{\prime} . \rho^{\prime}=x_{0} . f . \rho^{\prime}=X_{0}$. $\rho$.f. Thus, $y \notin x_{0} . \rho$, so that $Y \cap X_{0} . \rho=$ $\emptyset$. Since $Y \subseteq X . \rho=x_{0} . \rho \cup\left(X-x_{0}\right) . \rho$, we have $Y \subseteq\left(X-x_{0}\right) . \rho$. Therefore, $X-X_{0}$ dominates $Y$, which is a contradiction.

Suppose that $y^{\prime} \in Y_{0}^{\prime}$. Then, $Y_{0}^{\prime} \subseteq Y^{\prime}$ implies $y^{\prime} \in\left(X^{\prime}-x_{0}^{\prime}\right) . \rho^{\prime}$, which means that there exists $z^{\prime} \in X^{\prime}-x_{0}^{\prime}$ such that $y^{\prime} \in z^{\prime} . \rho^{\prime}$. This yields $z^{\prime} \in y^{\prime} . \rho^{\prime}=y . f . \rho^{\prime}=y . \rho . f$. Thus, there exists a point $z \in y . \rho$ such that $z^{\prime}=z . f$. Therefore, $y \in z . \rho$ and we have $z \in Y . \rho$ and $z . f=z^{\prime} \in X . f$. Hence, $z \in X$ by the assumption of the statement. But we also have $z \neq x_{0}$ because, otherwise, $z . f=x_{0}^{\prime} \neq z^{\prime}$, which is a contradiction. Thus, $z \in X-x_{0}$ so that $y \in\left(X-x_{0}\right)$. $\rho$. Again, we have shown that $Y \subseteq\left(X-x_{0}\right) . \rho$, i.e., that $X-x_{0}$ dominates $Y$, which is a contradiction. This proves the statement.

In Figure 6, only the minimal dominating sets aef, agh, bef and $c g$ satisfy the condition of Proposition 3.15. Each of them is mapped onto one of the minimal generating sets of $S^{\prime}$.

Reducing networks to simpler forms by strong homomorphisms provides an alternative method of approaching some of the classic problems in domination theory. However, it should be emphasized that "minimal" in domination theory means minimal cardinality whereas we use "minimal" in the set inclusion sense. Nevertheless, only sets that are minimal in the set inclusion sense can have minimal cardinality. So, network reduction by strong homomorphisms can still yield some insight into these classic problems.

Acknowledgement. The first author acknowledges support by the Ministry of Education, Youth and Sports of the Czech Republic from the National Programme of Sustainability (NPU II) project "IT4Innovations Excellence in Science - LQ1602". 


\section{References}

[1] Agnarsson, G. and Greenlaw, R. Graph Theory: Modeling, Applications and Algorithms (Prentice Hall, Upper Saddle River, NJ, 2007).

[2] Bourqui R., Gilbert, F., Simonetto, P., Zaidi, F., Sharan, U. and Jourdan, F. Detecting structural changes and command hierarchies in dynamic social networks, in: 2009 Advances in Social Network Analysis and Mining, 83-88 (Athens, Greece, 2009).

[3] Godsil, Ch. and Royle, G. Algebraic Graph Theory (Springer, New York, 2001).

[4] Harary, F. Graph Theory (Addison-Wesley, 1969).

[5] Haynes, T.W., Hedetniemi, S.T. and Slater, P.J. (editors) Domination in Graphs, Advanced Topics (Marcel Dekker, New York, 1998).

[6] Haynes, T.W., Hedetniemi, S.T. and Slater, P.J. Fundamentals of Domination in Graphs (Marcel Dekker, New York, 1998).

[7] Jankovic, D. and Hamlett, T.R. New topologies from old via ideals, Amer. Math. Monthly 97 (4), 295-310, 1990

[8] Koshevoy, G.A. Choice functions and abstract convex geometries, Mathematical Social Sciences, 38 (1), 35-44, 1999.

[9] McKee, T.A. and McMorris, F.R. Topics in Intersection Graph Theory, SIAM Monographs on Discrete Mathematics and Applications, Society for Industrial and Applied Mathematics (Philadelphia, PA, 1999).

[10] Monjardet, B. Closure operators and choice operators: a survey, in: Fifth International Conference on Concept Lattices and their Applications (Montpellier, France, 2007).

[11] Monjardet, $\mathrm{M}$ and Raderinirina, V. The duality between the antiexchange closure operators and the path independent choice operators on a finite set, Mathemetical Social Sciences 41(2), 131-150, 2001.

[12] Newman, M.E.J. The structure of function of complex networks, SIAM Review 45, 167-256, 2003.

[13] Oystein Ore. Mappings of closure relations, Annals of Math. 47 (1), 56-72, 1946.

[14] Pfaltz, J.L. and Šlapal, J. Transformations of discrete closure systems, Acta Math. Hungar. 138 (4), 386-405, 2013.

[15] Pfaltz, J.L. Closure lattices, Discrete Mathematics 154, 217-236, 1996.

[16] Pfaltz, J.L. Logical implication and causal dependency, in: Schärfe, H., Hitzler, P. and Ohrstrom, P. (editors) Conceptual Structures: Inspiration and Application, Lecture Notes in Artificial Intelligence 4068, 145-157, 2006.

[17] Pfaltz, J.L. Establishing logical rules from empirical data, Intern. Journal on Artificial Intelligence Tools 17 (5), 985-1001, 2008.

[18] Pfaltz, J.L. Mathematical continuity in dynamic social networks, in: Datta, A., Shulman, S., Zheng, B., Lin, S., Sun, A. and Lim, E.-P (editors) Third International Conference on Social Informatics 2011, Lecture Notes Comput. Sci. 6984, 36-50, 2011.

[19] Šlapal, J. Direct arithmetics of relational systems, Publ. Math. Debrecen 38, 39-48, 1991.

[20] Šlapal, J. Convenient closure operators on $\mathbb{Z}^{2}$, in: Wiederhold, P. and Barneva, R.P. (editors) Combinatorial Image Analysis, Lecture Notes Comput. Sci. 5852, 425-436, 2009.

[21] Šlapal, J. A Galois correspondence for digital topology, in: Denecke, K., Erné, M. and Wismath, S.L. (editors) Galois Connections and Applications, 413-424 (Kluwer Academic Publishers, Dordrecht, 2004). 
